\title{
Instability of channel flows of elastic liquids having continuously stratified properties
}

\author{
H.J Wilson ${ }^{1}$, J.M. Rallison* \\ Department of Applied Mathematics and Theoretical Physics, University of Cambridge, Silver Street, \\ Cambridge CB3 9EW, UK
}

Received 24 September 1998; received in revised form 9 November 1998

\begin{abstract}
This paper investigates inertialess channel flow of elastic liquids having continuously stratified constitutive properties. We find that an Oldroyd-B fluid having a sufficiently rapid normal stress variation shows instability. The mechanism is the same as for the two-fluid co-extrusion instability that arises when elasticity varies discontinuously. We find, using numerical and asymptotic methods, that this mechanism is opposed by convective effects, so that as the scale over which the elastic properties vary is increased, the growth rate is reduced, and finally disappears. A physical explanation for the stabilisation is given.

Regarding an Oldroyd-B fluid as a suspension of Hookean dumbbells, we show that a sufficiently steep variation in dumbbell concentration (with attendant rapid changes in both viscosity and elasticity) will provide an instability of the same kind.

Finally we show that Lagrangian convection of material properties (either polymer concentration or relaxation time) is crucial to the instability mechanism. A White-Metzner fluid having identical velocity and stress profiles in a channel flow is found to be stable. The implications for extrudate distortion, and constitutive modelling are briefly discussed. (C) 1999 Elsevier Science B.V. All rights reserved.
\end{abstract}

Keywords: Co-extrusion; Core-annular flow; Long wave; Instability; Oldroyd-B; White-Metzner; Normal stresses

\section{Introduction}

Purely elastic instabilities of homogeneous Oldroyd-B fluids are known to occur in flows having curved streamlines [1] and in plane parallel flows of co-extruded fluids where the viscosity is constant but there is a discontinuity in normal stress [2]. We do not know of any case in which a plane parallel flow of a fluid whose elastic properties are continuously stratified across the channel, has been shown

\footnotetext{
* Corresponding author. Fax: +44 1223337918.

${ }^{1}$ Current address: Department of Chemical Engineering, University of Colorado, Boulder, CO 80309-0424, USA.
} 
to be unstable. The primary purpose of this paper is to show that such an instability can occur, and to identify the circumstances in which it will do so.

A strong suggestion that such an instability could arise is given by the physical explanation of longwave instabilities for co-extruded elastic liquids provided by Hinch et al. [3]. The idea is that a perturbation of the interface between two different fluids exposes the jump in normal stress at the interface, which drives a flow. One might guess, then, that if a variation in normal stress occurs over a thin enough region, the same mechanism will generate an instability in a continuously stratified fluid. Indeed, at first sight this long-wave mechanism suggests that even a Poiseuille flow of an Oldroyd-B fluid with uniform elastic properties will be unstable, because the cross-stream variation of normal stress due to the variation of the shear-rate across the channel appears rapid to a long-wave disturbance. However, such a flow is stable (see, for example, [4] or [5]).

The long-wave argument above is, in general, erroneous for a fluid with continuously varying properties, because it fails to take proper account of convective effects. Consider pressure driven flow with centreline velocity $U_{0}$ in a channel of half-width $L$, sketched in Fig. 1. As shown in [3], in a flow with a fluid-fluid interface there is a long-wave instability, i.e. a disturbance for which the wavenumber $k L^{-1}$ is small. The disturbance is convected with velocity $u$, the streamwise velocity at the interface, and the growth is a second-order effect. Thus the complex frequency $\omega$ is

$$
\omega=k u / L+\mathrm{i} \sigma k^{2}+\mathrm{O}\left(k^{3}\right),
$$

where $\sigma$ is a constant (with dimensions of frequency), independent of $k$, having positive real part. The disturbance grows, on linear theory, on a timescale $\left(\sigma k^{2}\right)^{-1}$.

Now consider how this argument is modified if, instead, the fluid properties vary over a finite distance $q L$, where $q \ll 1$. The base-state shear-rate within this region is of order $U_{0} / L$ and so different fluid layers are convected at velocities which differ by a quantity of order $q U_{0}$. Thus on the growth timescale different layers generate perturbation elastic stresses that drift spatially out of phase with each other by a distance of order $\left(\sigma k^{2}\right)^{-1} q U_{0}$. In order for their phase difference not to exceed a wavelength we need $\left(\sigma k^{2}\right)^{-1} q U_{0}<k^{-1} L$, and therefore $q U_{0}(\sigma L)^{-1} \lesssim k$. Very long waves with $k<q U_{0}(\sigma L)^{-1}$ cannot be normal modes of the system because of the destructive interference between different layers of the blurred interface. Nevertheless, provided $q$ is small enough, disturbances for which $1 \gg k>q U_{0}(\sigma L)^{-1}$ will remain unstable.

In Section 3, we show that channel flows of elastic liquids having continuously varying elasticity are indeed subject to such instabilities. For the (somewhat artificial) model problem we have chosen, the fluid viscosity is a constant, but the elastic properties, defined by a Weissenberg number $W$, have a rapid variation. For this case both long-wave asymptotic (Section 3.1) and numerical (Section 3.2) solutions are found.

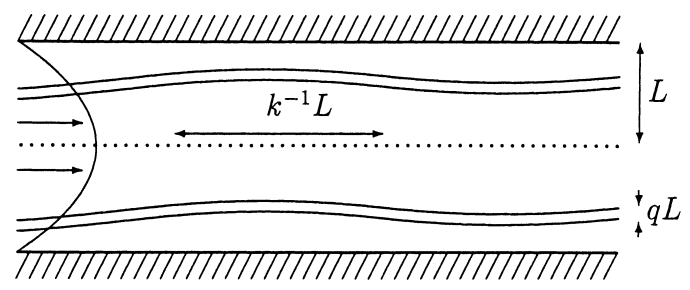

Fig. 1. Flow geometry. 
A more realistic case, amenable to experimental investigation, is considered in Section 4, where the concentration of polymer, $C$, varies rapidly within the channel. The new complication is that since the base-state viscosity varies rapidly, so does the velocity gradient. We first perform a calculation for the two-fluid case, in which the $C$-profile is discontinuous, and then give numerical results for the continuous profile analogous to that of Section 3. The extension to the case in which both $C$ and $W$ are stratified is straightforward.

Finally, in Section 5, we establish that the Lagrangian convection of material properties (either a polymer time constant or a concentration) is an essential ingredient for the development of this 'coextrusion' instability. In other words, we claim that a different fluid, in which material properties are not convected, may display the same base state velocity profile and stress distribution, and yet the unstable 'co-extrusion' mode may be absent.

We demonstrate this possibility by comparing a White-Metzner fluid, in which material properties depend on shear-rate, with the Oldroyd-B formalism, in which they are Lagrangian properties. We define two fluids having identical steady profiles of velocity and stress, and determine the effect of the model on the stability spectrum. Only the Oldroyd fluid shows instability.

Conclusions are given in Section 6, along with a discussion of the implications for constitutive modelling.

\section{Channel flow of a continuously stratified Oldroyd-B fluid}

We consider in this section the instability of an Oldroyd-B fluid whose properties vary continuously across a channel, for the flow sketched in Fig. 1.

It is convenient to scale lengths by the half-width of the channel, $L$; velocities by that on the centreline of the channel, $U_{0}$; and stresses by $U_{0} \mu / L$, where $\mu$ is the solvent viscosity.

The flow is inertialess and incompressible so that

$$
\begin{aligned}
& \nabla \cdot \boldsymbol{u}=0, \\
& \nabla \cdot \boldsymbol{\sigma}=0 .
\end{aligned}
$$

\subsection{Constitutive equation}

In non-dimensional form the constitutive equation may be written ${ }^{2}$

$$
\boldsymbol{\sigma}=-p \boldsymbol{I}+2 \boldsymbol{E}+\frac{C}{W} \boldsymbol{A}
$$

where $\boldsymbol{E}$ is the rate of strain tensor, and $\boldsymbol{A}$ evolves according to

$$
\stackrel{\nabla}{\boldsymbol{A}}=-\frac{1}{W}(\boldsymbol{A}-\boldsymbol{I}) .
$$

\footnotetext{
${ }^{2}$ The UCM equation, obtained in practice by ignoring the $\boldsymbol{E}$ term in Eq. (4) and setting $C=1$, may be obtained here by the limit $C \rightarrow \infty$.
} 
There are two parameters: $W$ and $C$, representing respectively the relaxation time and the concentration of polymer. They are defined as

$$
\begin{aligned}
& W=\frac{\lambda_{\mathrm{M}}(\boldsymbol{\eta}) U_{0}}{L}, \\
& C=\frac{\lambda_{\mathrm{M}}(\boldsymbol{\eta}) G_{\mathrm{M}}(\boldsymbol{\eta}) U_{0}^{2}}{L^{3} \mu},
\end{aligned}
$$

where $\lambda_{\mathrm{M}}$ is the relaxation time of the material and $G_{\mathrm{M}}$ is its elastic modulus.

For an inhomogeneous Oldroyd-B fluid either $C$ or $W$ is a material function, i.e. a function of the Lagrangian variable $\boldsymbol{\eta}(\boldsymbol{x}, t)$ that labels each material point. Because $\boldsymbol{\eta}$ is a material property

$$
\frac{\mathrm{D} \boldsymbol{\eta}}{\mathrm{D} t}=0
$$

This then implies that $\mathrm{D} C / \mathrm{D} t=0$ and $\mathrm{D} W / \mathrm{D} t=0$.

In investigating the stability of a steady planar channel flow, this (vector) description may be simplified by identifying each material point by its (scalar) position $y=\eta$ in the absence of any perturbation. In other words, we imagine that in the absence of any perturbation the continuously stratified fluid consists of many layers labelled by the Lagrangian coordinate $\eta$, for each of which the polymer concentration $C$, and relaxation time $W$ may be different.

\subsection{Base flow}

By symmetry, we consider only the upper half-channel $0 \leq y \leq 1$. The non-dimensionalisation fixes the driving pressure gradient, $G$. Denoting cross-channel differentiation, that is differentiation with respect to $y$, by a prime, we have boundary conditions $U(1)=0, U(0)=1$, and $U^{\prime}(0)=0$. The base state quantities become

$$
\begin{aligned}
& \boldsymbol{U}=(U(y), 0), \\
& \boldsymbol{A}=\left(\begin{array}{lc}
1+2 W^{2} U^{\prime 2} & W U^{\prime} \\
W U^{\prime} & 1
\end{array}\right),
\end{aligned}
$$

and, to within an additive constant the pressure is given as

$$
P=C / W+G x
$$

Finally,

$$
\Sigma=\left(\begin{array}{lc}
-G x+2 C W U^{\prime 2} & G y \\
G y & -G x
\end{array}\right) .
$$

Solving the $x$-momentum equation, the velocity $U(y)$ is given as

$$
U=1+G \int_{0}^{y} \frac{\eta}{1+C} d \eta
$$


and hence

$$
G=\frac{-1}{\int_{0}^{1} \eta /(1+C) d \eta} .
$$

In the particular case in which $C$ is a constant, the steady solution to the equations of motion is Poiseuille flow

$$
\boldsymbol{U}=\left(1-y^{2}, 0\right)
$$

The pressure gradient is then

$$
G=-2(1+C),
$$

and the other base state quantities are therefore

$$
\begin{aligned}
\boldsymbol{A} & =\left(\begin{array}{cc}
1+8 W^{2} y^{2} & -2 W y \\
-2 W y & 1
\end{array}\right), \\
\Sigma & =\left(\begin{array}{ll}
-P+C / W+8 C W y^{2} & -2(1+C) y \\
-2(1+C) y & -P+C / W
\end{array}\right), \\
P & =C / W-2(1+C) x .
\end{aligned}
$$

\subsection{Perturbation flow}

We impose a perturbation to the base state by adding an infinitesimal displacement $\zeta$ of a fluid particle in the cross-channel direction, so that a particle whose base-state position was $y=\eta$ moves to position $y=\eta+\zeta(\eta) \mathrm{e}^{\mathrm{i} k x-\mathrm{i} \omega t}$. Because $\zeta$ is small, we may write, correct to linear terms

$$
\eta(x, y, t)=y-\zeta(y) \mathrm{e}^{\mathrm{i} k x-\mathrm{i} \omega t}
$$

We denote perturbation quantities by lower case letters $(A+a, \Sigma+\sigma, W+w, C+c)$, and linearise about the base state.

Conservation of mass, $\{\boldsymbol{\nabla}\} \cdot \boldsymbol{u}=0$, becomes

$$
\mathrm{i} k u+v^{\prime}=0
$$

and we use a streamfunction $\psi(y)$ such that

$$
u=\psi^{\prime} \text { and } v=-\mathrm{i} k \psi
$$

to satisfy this requirement automatically.

The perturbation momentum equations become

$$
\begin{aligned}
& \mathrm{i} k \sigma_{11}+\sigma_{12}^{\prime}=0, \\
& \mathrm{i} k \sigma_{12}+\sigma_{22}^{\prime}=0 .
\end{aligned}
$$


The perturbation evolution equations give

$$
\begin{aligned}
& \sigma_{11}=-p+2 \mathrm{i} k \psi^{\prime}+\frac{C}{W} a_{11}+\frac{c}{W} A_{11}-\frac{C w}{W^{2}} A_{11}, \\
& \sigma_{12}=\psi^{\prime \prime}+k^{2} \psi+\frac{C}{W} a_{12}+\frac{c}{W} A_{12}-\frac{C w}{W^{2}} A_{12}, \\
& \sigma_{22}=-p-2 \mathrm{i} k \psi^{\prime}+\frac{C}{W} a_{22}+\frac{c}{W}-\frac{C w}{W^{2}} \\
& \left(-\mathrm{i} \omega+\mathrm{i} k U+\frac{1}{W}\right) a_{11}=\mathrm{i} k \psi A_{11}^{\prime}+2 A_{12} \psi^{\prime \prime}+2 A_{11} \mathrm{i} k \psi^{\prime}+2 U^{\prime} a_{12}+\frac{w}{W^{2}}\left(A_{11}-1\right), \\
& \left(-\mathrm{i} \omega+\mathrm{i} k U+\frac{1}{W}\right) a_{12}=\mathrm{i} k \psi A_{12}^{\prime}+\psi^{\prime \prime}+A_{11} k^{2} \psi+U^{\prime} a_{22}+\frac{w}{W^{2}} A_{12}, \\
& \left(-\mathrm{i} \omega+\mathrm{i} k U+\frac{1}{W}\right) a_{22}=-2 \mathrm{i} k \psi^{\prime}+2 A_{12} k^{2} \psi
\end{aligned}
$$

The streamline coordinate is a material quantity, so

$$
-\mathrm{i} k \psi-(-\mathrm{i} \omega+\mathrm{i} k U) \zeta=0,
$$

and the perturbations to $C$ and $W$ are then given as

$$
\begin{aligned}
& c=-\zeta C^{\prime}, \\
& w=-\zeta W^{\prime}
\end{aligned}
$$

The system Eqs. (20)-(30) is a fourth-order differential equation for $\psi$, and so we need four boundary conditions.

The no-slip, no-penetration boundary condition gives

$$
\psi(1)=\psi^{\prime}(1)=0 \text {. }
$$

As we are considering only the upper half-channel, the remaining boundary conditions are to be placed on the centreline $y=0$. If the perturbation is sinuous, $\psi$ must be an even function of $y$. Alternatively, for a varicose perturbation, $\psi$ is odd. We have, therefore,

$$
\begin{aligned}
& \text { Sinuous } \psi^{\prime}(0)=\psi^{\prime \prime \prime}(0)=0, \\
& \text { Varicose } \psi(0)=\psi^{\prime \prime}(0)=0 .
\end{aligned}
$$

The condition that we are looking for a nontrivial solution for $\psi$ provides the over-determination required to determine the eigenvalue $\omega$. The dispersion relation $\omega(k)$ is then defined by Eqs. (20)-(32).

\subsection{Alternative form of the equations}

A mathematically unfortunate, and physically inappropriate feature of the equations in Section 2.3 arises in the important limiting case where two distinct fluids are present. Either $C$ or $W$ is then a 
discontinuous function, and the equations above include products of generalised functions, which are awkward to deal with.

Motivated by a physical understanding of the way in which the instability operates [3], we are led to rewrite the equations by splitting the stresses, stretches and pressure into two parts: $\sigma=\sigma^{\text {adv }}+\sigma^{\text {dyn }}$, $a=a^{\text {adv }}+a^{\text {dyn }}, p=p^{\text {adv }}+p^{\text {dyn }}$, where $x^{\text {adv }}$ is the part of quantity $X$ that arise from advection of $C$ or $W$ by the streamline displacement alone. These advected contributions are given as

$$
\begin{aligned}
& p^{\mathrm{adv}}=c / W+w\left(-C / W^{2}\right), \\
& a_{22}^{\mathrm{adv}}=0 \\
& a_{12}^{\mathrm{adv}}=c\left(-W U^{\prime} /(1+C)\right)+w U^{\prime}, \\
& a_{11}^{\mathrm{adv}}=c\left(-4 W^{2} U^{\prime 2} /(1+C)\right)+w\left(4 W U^{2}\right), \\
& \sigma_{22}^{\mathrm{adv}}=\sigma_{12}^{\mathrm{adv}}=0 \\
& \sigma_{11}^{\mathrm{adv}}=c\left(2 W U^{\prime 2}(1-C) /(1+C)\right)+w\left(2 C U^{\prime 2}\right) .
\end{aligned}
$$

The remaining dynamic parts are driven by the advected perturbations. The momentum equations become (omitting the superscript ${ }^{\text {dyn }}$ )

$$
\begin{aligned}
& \mathrm{i} k \sigma_{11}+\sigma_{12}^{\prime}=2 \mathrm{i} k \zeta G^{2} y^{2}\left\{W^{\prime} C /(1+C)^{2}+W C^{\prime}(1-C) /(1+C)^{3}\right\}, \\
& \mathrm{i} k \sigma_{12}+\sigma_{22}^{\prime}=0 .
\end{aligned}
$$

The effect of the splitting is to introduce a 'body force' into the perturbation momentum Eq. (39), arising from the advection of the normal stress in the base state.

The constitutive equations become

$$
\begin{aligned}
& \sigma_{11}=-p+2 \mathrm{i} k \psi^{\prime}+\frac{C}{W} a_{11} \\
& \sigma_{12}=\psi^{\prime \prime}+k^{2} \psi+\frac{C}{W} a_{12}-\zeta G y C^{\prime} /(1+C)^{2} \\
& \sigma_{22}=-p-2 \mathrm{i} k \psi^{\prime}+\frac{C}{W} a_{22} \\
& \left(-\mathrm{i} \omega+\mathrm{i} k U+\frac{1}{W}\right) a_{11}=\mathrm{i} k \psi \frac{4 W^{2} G^{2} y}{(1+C)^{2}}+2 A_{12} \psi^{\prime \prime}+2 A_{11} \mathrm{i} k \psi^{\prime}+2 U^{\prime} a_{12}-2 A_{12} \zeta G y C^{\prime} /(1+C)^{2} \\
& \left(-\mathrm{i} \omega+\mathrm{i} k U+\frac{1}{W}\right) a_{12}=\mathrm{i} k \psi \frac{W G}{(1+C)}+\psi^{\prime \prime}+A_{11} k^{2} \psi+U^{\prime} a_{22}-\zeta G y C^{\prime} /(1+C)^{2} \\
& \left(-\mathrm{i} \omega+\mathrm{i} k U+\frac{1}{W}\right) a_{22}=-2 \mathrm{i} k \psi^{\prime}+2 A_{12} k^{2} \psi
\end{aligned}
$$


and the kinematic condition is

$$
(-\mathrm{i} \omega+\mathrm{i} k U) \zeta=-\mathrm{i} k \psi \text {. }
$$

Eqs. (39)-(49) are easier to manipulate than those of Section 2.3 because of the limited number of occurrences of derivatives of $C$ and $W$, and it is now possible to proceed to the discontinuous two-fluid limit. Taken together with the unchanged boundary conditions Eqs. (31-32), the new equations define $\omega(k)$.

\section{Instability of a constant viscosity Oldroyd-B fluid}

We now consider the case in which $C$ is a constant, for which the viscosity throughout the channel is $1+C$ and is constant, the velocity profile is parabolic, and the base state quantities are given by Eqs. (15)-(19). Perturbation quantities are governed by Eqs. (39)-(47), in which all terms involving $C^{\prime}$ are neglected.

The advantage of the transformation in Section 2.4 is now apparent: the form of these equations is identical to those for which $W$ is a constant except for the appearance of $W^{\prime}(y)$ in the momentum Eq. (39).

It remains to choose the profile $W(\eta)$. We consider a symmetric profile so that $W(\eta)=W(-\eta)$. A convenient choice for $\eta>0$ is then

$$
W=\bar{W}+\frac{\Delta W}{\pi}\{\arctan (\eta-\kappa) / q\},
$$

in which $W$ changes by an amount $\Delta W$ across a layer, centred at $\eta=\kappa$, of dimensionless width $q$. In the limit $q \rightarrow 0$

$$
\begin{array}{ll}
W=\bar{W}+\Delta W / 2, & \eta>\kappa, \\
W=\bar{W}-\Delta W / 2, \quad \eta<\kappa,
\end{array}
$$

and we recover the two-fluid co-extrusion problem of [3], for which integration of Eq. (39) across the interface yields the continuity condition

$$
\left[\sigma_{12}\right]=8 \mathrm{i} k C \kappa^{2} \Delta W \zeta(\kappa),
$$

where $y=\kappa$ is the position of the interface, and square brackets denote a jump across it.

\subsection{Long-wave asymptotics}

The physical argument in Section 1 suggests that the most likely candidate for a long-wave $(k \rightarrow 0)$ instability is a layer having width of order $k$. In consequence, we consider the distinguished limit in which $q=\tilde{q} k$ and $k \rightarrow 0$ with $\tilde{q}$ fixed. In that case, except in a layer of size $k$ around $y=\kappa, W$ takes constant values $\bar{W}+\Delta W$ for $y>\kappa$, and $\bar{W}-\Delta W$ for $y<\kappa$. As a result, the outer flow has the dynamics of two co-extruded Oldroyd-B fluids with matched viscosities $1+C$.

There are two regions (sketched in Fig. 2): the outer region, consisting of the main body of both fluids, where the Weissenberg number is constant and the equations are those of uniform Oldroyd-B fluids; and the interior of the varying layer, in which the $W^{\prime}$ term plays a large part. 


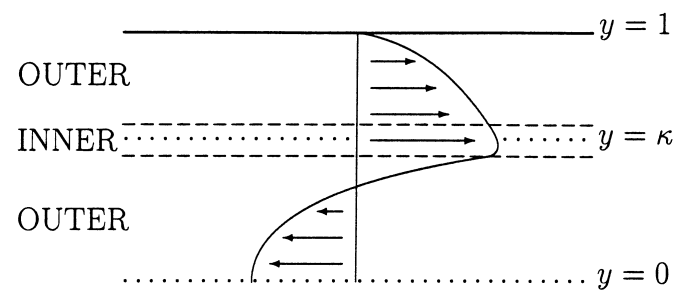

Fig. 2. Sketch of the perturbation flow profile for a varicose disturbance. Note the discontinuity in the slope $u^{\prime}$ across the inner region, arising from $\left[\sigma_{12}\right]$.

So far as the outer flow is concerned we may impose, by Eq. (39), a discontinuity [ $\left.\sigma_{12}\right]$ in tangential stress at $y=\kappa$. The magnitude of this discontinuity (which may be a complex number) is as yet unknown, and must be determined by analysis of the thin layer itself. We anticipate that the frequency of the disturbance will continue to have the form of Eq. (1)

$$
\omega=k u+\mathrm{i} \sigma k^{2},
$$

and thus the Deborah number, $\omega \tau$, associated with the perturbation is of order $k$ and so is small. In consequence, the perturbation elastic stresses respond quasi-statically to the flow, so giving rise to a Newtonian stress having shear viscosity $(1+C)$.

\subsubsection{Outer flow}

Because the disturbance wavelength is much longer that the channel width, lubrication theory can be used: the leading order perturbation flow is in the $x$-direction, the pressure across the channel is uniform, and so the flow profile is parabolic for a varicose mode, or linear if the mode is sinuous.

The velocity must be continuous across $y=\kappa$, and the tangential stress balance across the interface gives

$$
\left[(1+C) u^{\prime}\right]_{\kappa-}^{\kappa+}=\left[\sigma_{12}\right]
$$

For a sinuous mode, the pressure gradient must be an odd function and is thus zero. We then obtain

$$
\begin{array}{ll}
u(y)=\frac{\left[\sigma_{12}\right]\{-(1-\kappa) y\}}{1+C}, & 0<y<\kappa, \\
u(y)=\frac{\left[\sigma_{12}\right]\{-\kappa(1-y)\}}{1+C}, & \kappa<y<1,
\end{array}
$$

and mass conservation gives the streamfunction $\psi$ at the interface as

$$
\psi(\kappa)=-\int_{\kappa}^{1} u \mathrm{~d} y=\frac{\left[\sigma_{12}\right] f_{\mathrm{s}}(\kappa)}{2(1+C)}
$$

where

$$
f_{\mathrm{s}}(\kappa)=\kappa(1-\kappa)^{2}
$$


For a varicose mode the perturbation pressure gradient along the channel must be such as to make the perturbation volume flux along the channel vanish. After some algebra, we obtain

$$
\begin{aligned}
& u(y)=\frac{\left[\sigma_{12}\right]\left\{-(1-\kappa)(1-3 \kappa)-3(1-\kappa)(1+\kappa) y^{2}\right\}}{4(1+C)}, \quad 0<y<\kappa, \\
& u(y)=\frac{\left[\sigma_{12}\right]\left\{2\left(3 \kappa^{2}-1\right)(y-1)-\frac{3}{4}(1-\kappa)(1+\kappa)(y-1)^{2}\right\}}{4(1+C)}, \quad \kappa<y<1,
\end{aligned}
$$

and

$$
\psi(\kappa)=\frac{\left[\sigma_{12}\right] f_{\mathrm{v}}(\kappa)}{2(1+C)},
$$

where

$$
f_{\mathrm{v}}(\kappa)=\frac{1}{2} \kappa(1-\kappa)^{2}\left(\kappa^{2}+2 \kappa-1\right) .
$$

A typical profile for the perturbation flow is sketched in Fig. 2.

Thus, in either case

$$
\psi(\kappa)=\frac{\left[\sigma_{12}\right] f(\kappa)}{2(1+C)},
$$

where $f=f_{\mathrm{s}}$ for a sinuous mode and $f=f_{\mathrm{v}}$ for a varicose mode.

\subsubsection{Inner solution}

Within the thin layer where the fluid properties change, the relevant length-scale for $y$ is $k$. Because $k$ is small, the pressure within the layer is constant, and the $x$-momentum equations, Eq. (39), reduces to

$$
(1+C) \psi^{\prime \prime \prime}-8 \mathrm{i} C k y^{2} W^{\prime} \zeta=0
$$

where (Eq. (28))

$$
\zeta=-\mathrm{i} k \psi /\left\{-\mathrm{i} \omega+\mathrm{i} k\left(1-y^{2}\right)\right\} .
$$

It is convenient to define a scaled coordinate for the layer. We write

$$
\xi=k^{-1}(y-\kappa) \text { with } \xi=\mathrm{O}(1) .
$$

Then from the expression (1) for $\omega$ we have

$$
\omega=k\left(1-\kappa^{2}\right)+\mathrm{i} \sigma k^{2},
$$

where $\sigma$ might itself be complex, and then

$$
(1+C) \frac{\mathrm{d}^{3} \psi}{\mathrm{d} \xi^{3}}-8 \mathrm{i} C k^{2} \frac{\kappa^{2}}{\mathrm{i} \sigma+2 \kappa \xi} \psi(\kappa) \frac{\mathrm{d} W}{\mathrm{~d} \xi}=0 .
$$


It follows, as expected, that as $k \rightarrow 0, \psi$ and $\psi^{\prime}$ are continuous across the layer, and that $\psi$ has the value $\psi(\kappa)$ in Eq. (52). Furthermore, for self-consistency we must have

$$
\begin{aligned}
{\left[\sigma_{12}\right] } & =(1+C)\left[\psi^{\prime \prime}\right]_{-\infty}^{\infty}=(1+C) k^{-2}\left[\mathrm{~d}^{2} \psi / \mathrm{d} \xi^{2}\right]_{-\infty}^{\infty}=(1+C) k^{-2} \int_{-\infty}^{\infty} \frac{\mathrm{d}^{3} \psi}{\mathrm{d} \xi^{3}} \mathrm{~d} \xi \\
& =8 \mathrm{i} C \kappa^{2} \psi(\kappa) \int_{-\infty}^{\infty} \frac{\mathrm{d} W / \mathrm{d} \xi}{\mathrm{i} \sigma+2 \kappa \xi} \mathrm{d} \xi .
\end{aligned}
$$

Substituting for $\psi(\kappa)$ gives the dispersion relation for $\sigma$

$$
\int_{-\infty}^{\infty} \frac{\mathrm{d} W / \mathrm{d} \xi}{\mathrm{i} \sigma+2 \kappa \xi} \mathrm{d} \xi=\frac{1+C}{4 \mathrm{i} C \kappa^{2} f(\kappa)},
$$

where $f(\kappa)=f_{\mathrm{s}}(\kappa)$ for a sinuous mode, and $f(\kappa)=f_{\mathrm{v}}(\kappa)$ for a varicose mode.

For co-extrusion of two distinct fluids, $\mathrm{d} W / \mathrm{d} \xi=\Delta W \delta(\xi)$, where $\delta$ is a Dirac $\delta$-function, and thus

$$
\sigma=\sigma_{0} \equiv \Delta W \frac{4 C \kappa^{2} f(\kappa)}{1+C}
$$

and this agrees with [3].

If, on the other hand, we consider the continuous variation for $W$ given by Eq. (48), then

$$
\frac{\mathrm{d} W}{\mathrm{~d} \xi}=\frac{1}{\pi} \frac{\Delta W \tilde{q}}{\xi^{2}+\tilde{q}^{2}},
$$

and thus

$$
\frac{1}{\pi} \int_{-\infty}^{\infty} \frac{\tilde{q} \mathrm{~d} \xi}{\left(\tilde{q}^{2}+\xi^{2}\right)(\mathrm{i} \sigma+2 \kappa \xi)}=\frac{1}{\mathrm{i} \sigma_{0}} .
$$

This integral is easily evaluated by the calculus of residues, giving

$$
\begin{array}{cl}
\frac{1}{\mathrm{i} \sigma_{0}}=(\mathrm{i} \sigma+2 \mathrm{i} k \tilde{q})^{-1}, & \operatorname{Re}(\sigma)>0, \\
\frac{1}{\mathrm{i} \sigma_{0}}=(\mathrm{i} \sigma-2 \mathrm{i} k \tilde{q})^{-1}, & \operatorname{Re}(\sigma)<0 .
\end{array}
$$

We deduce that if $\left|\sigma_{0}\right|<2 \kappa \tilde{q}$ there are no acceptable modes of the kind sought, but that if $\left|\sigma_{0}\right|>2 \kappa \tilde{q}$ then

$$
|\sigma|=\left|\sigma_{0}\right|-2 \kappa \tilde{q} .
$$

All these estimates are confirmed by a full matched asymptotic expansion (see [6]).

Remembering that $\tilde{q}=q / k$, where $q$ is the width of the blurred interface, the growth rate $|\sigma| k^{2}$ of the disturbance may be written as

$$
|\sigma| k^{2}=|\Delta W| \frac{4 C \kappa^{2} f(\kappa)}{1+C} k^{2}-2 \kappa q k
$$


provided this quantity is positive, but no mode of this kind exists if it is negative. We deduce that very long waves $(k \rightarrow 0)$ cannot be normal modes, but that provided $q$ is small enough a long-wave unstable co-extrusion mode will still appear.

\subsection{Numerical results}

Eqs. (20)-(30) define a fourth-order ordinary differential equation for the unknown eigenvalue $\omega$. Away from the long-wave limit it is necessary to solve this equation numerically. The method used follows Ho and Denn [4] and involves shooting from $y=0$ to $y=1$, together with a Newton-Raphson iterative solution to determine $\omega$. We find it essential, as in [4], to orthonormalise solutions to the differential equation in order to maintain accuracy.

For very small values of $q$, the eigenvalues are identical to those for the two-fluid problem. As the width of 'blurring', $q$, increases, the eigenvalues become neutral. A set of results for a typical choice of parameters is shown in Fig. 3.

Increasing $\Delta W$ in Eq. (55) increases the largest value of $q$ for which the eigenvalue exists. This process cannot be continued indefinitely however, because as the elasticity contrast $\Delta W$ is increased, so is the elasticity of one or other fluid, and this fluid resists deformation (see Wilson and Rallison [7]). For $q>0.04$ no interfacial modes, stable or unstable, could be found for any choice of parameters.

As predicted, very long waves disappear as soon as $q \neq 0$. Only waves having $k>2 \kappa q /\left|\sigma_{0}\right|$ consistent with Eq. (55) can appear. Shorter waves having $k \sim 1$ survive longest and are the most unstable.

Note that this disturbance does not restabilise outside its unstable range: rather it ceases to exist. It can therefore be difficult to find by parameter continuation.

The disappearance or appearance of a mode must be associated with a singularity of the governing equations. In this case, the coefficient $-\mathrm{i} \omega+\mathrm{i} k U$ in Eq. (47) becomes zero at the point where the mode

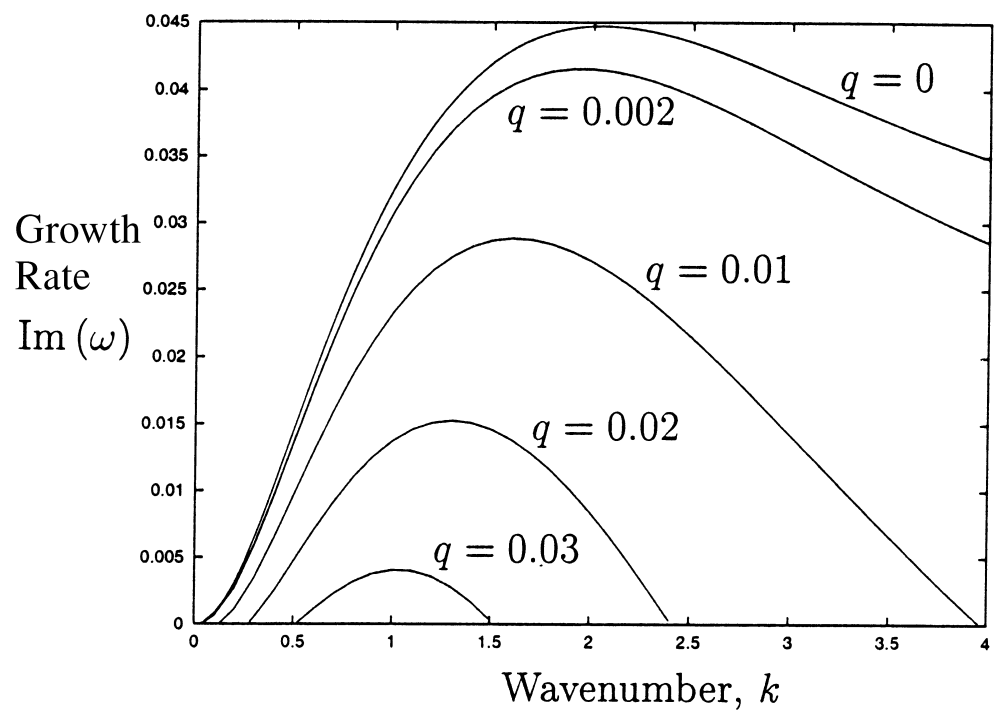

Fig. 3. Reduction of instability for increased 'blurring': $C=0.2, \bar{W}=2, \Delta W=4$. Sinuous mode. The curve for $q=0$ is the two-fluid case as in [7]. 
appears. This term arises from the material derivative $\mathrm{D} / \mathrm{D} t$, and is present only because $W$ is advected as a material quantity. We expect, therefore, that this mode should exist only for constitutive equations where there is advection of material properties. We return to this matter in Section 5.

\section{Flow of an Oldroyd-B fluid with varying polymer concentration}

In this section, we consider a fluid for which the concentration of polymer changes across the channel. On the assumption that the polymers are advected with the flow, the concentration is $C(\eta)$ where $\eta$ is a Lagrangian coordinate.

This fluid models a suspension of microscopic dumbbells for which the concentration $C$ varies. This concentration is purely advected only if cross-streamline diffusion is neglected. If such diffusion were to be taken into account, this could provide a stabilising mechanism (by increasing the 'blurring' of the interface). However, the mechanism for diffusion is Brownian motion, as is the mechanism for relaxation of the dumbbell. We have scaled our equations so that this relaxation time is of order unity, and so the time taken for a dumbbell to diffuse over its own diameter is also of order unity. The crosschannel diffusion rate thus scales as the square of the ratio of dumbbell size to channel width. We have already assumed that this ratio is small in order to use continuum mechanics; for a channel of $1 \mathrm{~cm}$ and dumbbells of size $1 \mu \mathrm{m}$, the ratio is $10^{-4}$, so the diffusion rate of order $10^{-8}$. We conclude that diffusion is unimportant here.

\subsection{Base state}

As shown in Section 2.2, the unidirectional channel flow profile is the solution of the differential equation

$$
U^{\prime}=G y /(1+C) .
$$

For this flow profile the remaining base state quantities are

$$
\begin{aligned}
& \boldsymbol{A}=\left(\begin{array}{lc}
1+2 W^{2} U^{\prime 2} & W U^{\prime} \\
W U^{\prime} & 1
\end{array}\right) . \\
& P=C / W+G x,
\end{aligned}
$$

and

$$
\Sigma=\left(\begin{array}{lc}
-G x+2 C W U^{\prime 2} & G y \\
G y & -G x
\end{array}\right) .
$$

In these expressions, the concentration $C$ may depend on $y$. In the two-fluid case where

$$
\begin{array}{ll}
C=C_{1}, & |y|>\kappa, \\
C=C_{2}, & |y|<\kappa,
\end{array}
$$




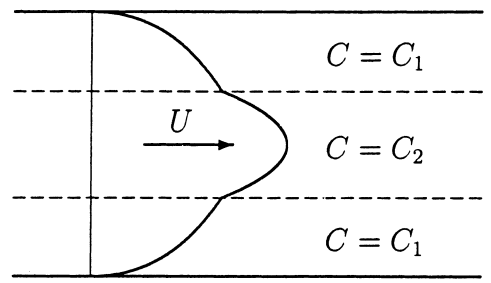

Fig. 4. Flow profile $U$ in the channel, for the case of two separate fluids in which the higher viscosity fluid is on the outside, $C_{1}>C_{2}$.

we obtain

$$
\begin{aligned}
& U=\frac{G\left(y^{2}-1\right)}{2\left(1+C_{1}\right)}, \quad|y|>\kappa \\
& U=\frac{G\left\{\left(1+C_{1}\right)\left(y^{2}-\kappa^{2}\right)-\left(1+C_{2}\right)\left(1-\kappa^{2}\right)\right\}}{2\left(1+C_{1}\right)\left(1+C_{2}\right)}, \quad|y|<\kappa \\
& G=\frac{-2\left(1+C_{1}\right)\left(1+C_{2}\right)}{\left(1+C_{1}\right) \kappa^{2}+\left(1+C_{2}\right)\left(1-\kappa^{2}\right)} .
\end{aligned}
$$

This is, of course, the Newtonian profile for two fluids of viscosity $1+C_{1}$ and $1+C_{2}$ respectively (sketched in Fig. 4 for a case where $C_{1}>C_{2}$ ). Note that it involves a discontinuity in $U^{\prime}$ at $y=\kappa$. There is additionally a discontinuity in $N_{1}$ of magnitude $2 W G^{2} \kappa^{2}\left[C /(1+C)^{2}\right]$ at the interface.

\subsection{Stability problem for two Oldroyd-B fluids}

\subsubsection{Governing equations}

Within each fluid separately, the governing equations are Eqs. (39)-(47), in which all terms involving the derivatives $C^{\prime}$ and $W^{\prime}$ are neglected, and with $C=C_{1}$ or $C_{2}$ as appropriate.

At the interface, $y=\kappa+\zeta \exp (\mathrm{i} k x-\mathrm{i} \omega t)$, we require continuity of $\boldsymbol{U}+\boldsymbol{u}$ and of the traction $(+\boldsymbol{\sigma}) \cdot \boldsymbol{n}$. These conditions become continuity at $y=\kappa$ of $\psi, \psi^{\prime}+\zeta U^{\prime}, \sigma_{12}-\mathrm{i} k \zeta \Sigma_{11}$ and $\sigma_{22}$. It is the appearance of the $\zeta U^{\prime}$ term here, involving the discontinuity of $U^{\prime}$, that differs from the flows considered in $[2,3,7,8]$.

\subsubsection{Long-wave limit}

In the limit of small $k$, we pose the series

$$
\omega=k \omega_{1}+\mathrm{i} k^{2} \sigma+\mathrm{O}\left(k^{3}\right),
$$

and expand all quantities in $k$. The full details of this analysis are given in [6]: we outline the principal features here.

\section{Sinuous mode.}

Using, for convenience, the notation

$$
g\left(\chi, \phi_{1}, \phi_{2}\right) \equiv \chi \phi_{1}+(1-\chi) \phi_{2},
$$


we obtain, at leading order, for a sinuous mode

$$
\psi_{0}(\kappa)=\frac{\left(1+C_{2}\right) \kappa(1-\kappa)^{2}\left(C_{1}-C_{2}\right) \zeta}{g\left(\kappa, 1+C_{1}, 1+C_{2}\right) g\left(\kappa^{2}, 1+C_{1}, 1+C_{2}\right)},
$$

and thus

$$
\omega_{1}=\frac{\left(1+C_{2}\right)\left(1-\kappa^{2}\right)}{g\left(\kappa^{2}, 1+C_{1}, 1+C_{2}\right)}+\frac{\left(1+C_{2}\right) \kappa(1-\kappa)^{2}\left(C_{1}-C_{2}\right)}{g\left(\kappa, 1+C_{1}, 1+C_{2}\right) g\left(\kappa^{2}, 1+C_{1}, 1+C_{2}\right)} .
$$

We note that the disturbance does not convect with the interface, as it does for the matched-viscosity case of [7] and Section 3. At this leading order, the fluids are Newtonian so we may check this result by comparing with the flow (in the same geometry) of two Newtonian fluids with different viscosities ${ }^{3}$

The growth rate, which appears at order $k^{2}$, is

$$
\sigma=\frac{1}{2} \kappa(\kappa-1)^{2} \frac{M_{0}\left[U^{\prime}\right]+\left[\Sigma_{11}\right]}{g\left(\kappa, 1+C_{1}, 1+C_{2}\right)}+\frac{1}{3}(\kappa-1)^{3} M_{1}\left[U^{\prime}\right]+\frac{1}{4}(\kappa-1)^{4} \frac{M_{1}\left[U^{\prime}\right]\left(1+C_{2}\right)}{g\left(\kappa, 1+C_{1}, 1+C_{2}\right)},
$$

where

$$
\begin{aligned}
M_{0}= & \frac{-W G\left(1+C_{2}\right)^{2}(\kappa-1)^{2}}{2 g\left(\kappa, 1+C_{1}, 1+C_{2}\right)^{2}}\left(\frac{C_{1}}{1+C_{1}}-\frac{C_{2}}{1+C_{2}}\right)-2 W G \kappa \\
& +\frac{2 W G \kappa g\left(\kappa,\left(1+C_{1}\right)^{2},\left(1+C_{2}\right)^{2}\right)}{g\left(\kappa, 1+C_{1}, 1+C_{2}\right)\left(1+C_{1}\right)\left(1+C_{2}\right)}, \\
M_{1}= & \frac{W G}{g\left(\kappa, 1+C_{1}, 1+C_{2}\right)} \frac{C_{1}\left(1+C_{2}\right)}{\left(1+C_{1}\right)^{2}},
\end{aligned}
$$

\footnotetext{
${ }^{3}$ Consider the channel flow of two Newtonian fluids with viscosities $\mu_{1}, \mu_{2}$. The perturbation to the interface exposes the jump in $U^{\prime}$ due to the viscosity difference, causing a discontinuity in velocity at the interface, and this generates a perturbation flux $\Delta Q$ given by
}

$$
\Delta Q=\int_{0}^{\kappa+\zeta} U_{2}^{\prime} \mathrm{d} y+\int_{\kappa+\zeta}^{1} U_{1}^{\prime} \mathrm{d} y+1=-\zeta\left[U^{\prime}\right]_{\kappa} .
$$

This extra flux must be balanced by an additional flow, and since for a sinuous mode this flow is linear in $y$, and the shear stress is continuous, the velocity profile is

$$
\begin{aligned}
& u(y)=\frac{-\zeta\left[U^{\prime}\right] \mu_{2}(y-1)}{\mu_{1} \kappa+\mu_{2}(1-\kappa)}, \quad y>\kappa, \\
& u(y)=\frac{-\zeta\left[U^{\prime}\right] \mu_{1} y}{\mu_{1} \kappa+\mu_{2}(1-\kappa)}, \quad y<\kappa .
\end{aligned}
$$

Mass conservation then gives

$$
\psi(\kappa)=\frac{1}{2}(\kappa-1)^{2} \frac{\zeta\left[U^{\prime}\right] \mu_{2}}{g\left(\kappa, \mu_{1}, \mu_{2}\right)},
$$

and hence as in Eq. (57)

$$
\omega=k U(\kappa)+k \frac{\mu_{2} \kappa(1-\kappa)^{2}\left(\mu_{1}-\mu_{2}\right)}{g\left(\kappa, \mu_{1}, \mu_{2}\right) g\left(\kappa^{2}, \mu_{1}, \mu_{2}\right)} .
$$


and the jump quantities are

$$
\begin{aligned}
& {\left[U^{\prime}\right]=G \kappa\left(\frac{1}{1+C_{1}}-\frac{1}{1+C_{2}}\right),} \\
& {\left[\Sigma_{11}\right]=2 W G^{2} \kappa^{2}\left(\frac{C_{1}}{\left(1+C_{1}\right)^{2}}-\frac{C_{2}}{\left(1+C_{2}\right)^{2}}\right) .}
\end{aligned}
$$

In these expressions, the pressure gradient, $G$, is given as

$$
G=\frac{-2\left(1+C_{1}\right)\left(1+C_{2}\right)}{g\left(\kappa^{2}, 1+C_{1}, 1+C_{2}\right)} .
$$

We note that the growth rate is proportional to the Weissenberg number $W$, indicating once again that it is the normal stress jump that drives the instability. As we would expect, the case $C_{1}=C_{2}$ where the fluids are identical is neutrally stable. We can make some progress in understanding these results by considering extreme values for $\kappa$.

In the limit $\kappa \rightarrow 0$, the growth rate becomes

$$
\sigma \sim-\frac{1}{3} \kappa W C_{1}\left[\frac{1}{1+C}\right]=\frac{1}{3} \kappa W\left(C_{1}-C_{2}\right) \frac{C_{1}}{\left(1+C_{1}\right)\left(1+C_{2}\right)},
$$

so the mode is unstable if $C_{1}>C_{2}$, i.e. if the large outer fluid has the higher viscosity.

As $\kappa \rightarrow 1$, we have

$$
\sigma \sim 4(\kappa-1)^{2} W \frac{\left(1+C_{2}\right)^{2}}{1+C_{1}}\left(\left[\frac{C}{(1+C)^{2}}\right]-\frac{C_{2}}{\left(1+C_{2}\right)}\left[\frac{1}{1+C}\right]\right)=4(\kappa-1)^{2} W\left(C_{1}-C_{2}\right) \frac{\left(1+C_{2}\right)}{\left(1+C_{1}\right)^{3}},
$$

which again indicates weak instability (at order $\left.(\kappa-1)^{2}\right)$ if $C_{1}>C_{2}$.

For intermediate values of $\kappa$, however, the sign of this growth rate is not obvious. The disturbance is stable for some triples $\left(\kappa, C_{1}, C_{2}\right)$ and unstable for others. For instance, at $\kappa=\frac{1}{2}$ we have the values shown in Table 1 , in which we observe that instability may occur when either $C_{1}>C_{2}$ or $C_{2}>C_{1}$, depending on the specific parameter values.

\section{Varicose mode}

For a varicose mode, the equations are similar. The leading-order growth rate has been calculated using 'Maple' [9]. The result is more complicated than the sinuous result of Eq. (58), and is not reproduced here.

Table 1

Growth rate $\sigma$ for sample values of $C_{1}$ and $C_{2}$ for a sinuous mode at $\kappa=\frac{1}{2}$

\begin{tabular}{llr}
\hline$C_{1}$ & $C_{2}$ & $\sigma$ \\
\hline 2 & 6 & $-0.037679 \mathrm{~W}$ \\
0 & 6 & $0.003390 \mathrm{~W}$ \\
6 & 2 & $0.052286 \mathrm{~W}$ \\
6 & 0 & $0.069442 \mathrm{~W}$ \\
\hline
\end{tabular}


However, we can examine the limits of small and large $\kappa$. For a slender inner fluid, $\kappa \rightarrow 0$, we have

$$
\sigma \sim \kappa^{3} W\left(C_{1}-C_{2}\right) \frac{\left(C_{2} C_{1}-2-C_{1}\right)}{\left(1+C_{2}\right)^{2}\left(1+C_{1}\right)},
$$

and even here the sign of $\sigma$ is not obvious.

We expect that, in the limit $\kappa \rightarrow 1$, the two interfaces are too far apart to feel each other's effect. The growth rates should be the same for sinuous and varicose modes. We indeed obtain the sinuous result above

$$
\sigma \sim 4(\kappa-1)^{2} W\left(C_{1}-C_{2}\right) \frac{\left(1+C_{2}\right)}{\left(1+C_{1}\right)^{3}} .
$$

Fig. 5 shows a plot of the long-wave growth rate $\sigma / W$ against interfacial position $\kappa$ for the illustrative case $C_{1}=6, C_{2}=0$. We see that the sinuous modes generally have the higher long-wave growth rate. However, it is possible for varicose modes alone to be unstable: for example, for small $\kappa$, if $C_{1}=0$ then the growth rate is $\sigma=2 \kappa^{3} W C_{2}\left(1+C_{2}\right)^{-2}$ for the varicose mode, while the sinuous mode is stable.

\subsubsection{Numerical results for general $k$}

The equations are solved using the numerical method described in Section 3.2, integrating from the wall and centre-line to the interface, and applying jump conditions there. Some typical plots of growth rate against wavenumber are shown in Figs. 6 and 7. The long-wave asymptote $\operatorname{Im}(\omega) \sim k^{2} \sigma$ may be checked against our analytic results. Where long waves are unstable, a wavelength comparable with

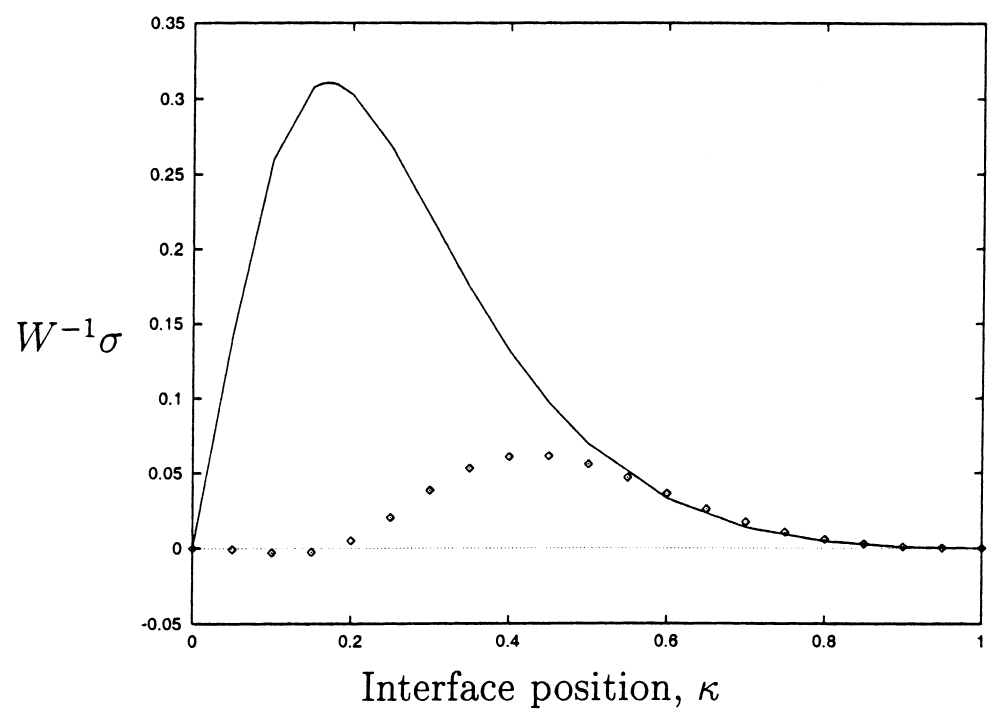

Fig. 5. Plot of the growth rate of long waves against interface position, $\kappa$, for the representative case $C_{1}=6, C_{2}=0$. The solid line is for sinuous modes and the points for varicose modes. The sinuous modes usually have higher growth rate, and are always unstable, whereas the varicose modes are stable for $\kappa<0.175$. 


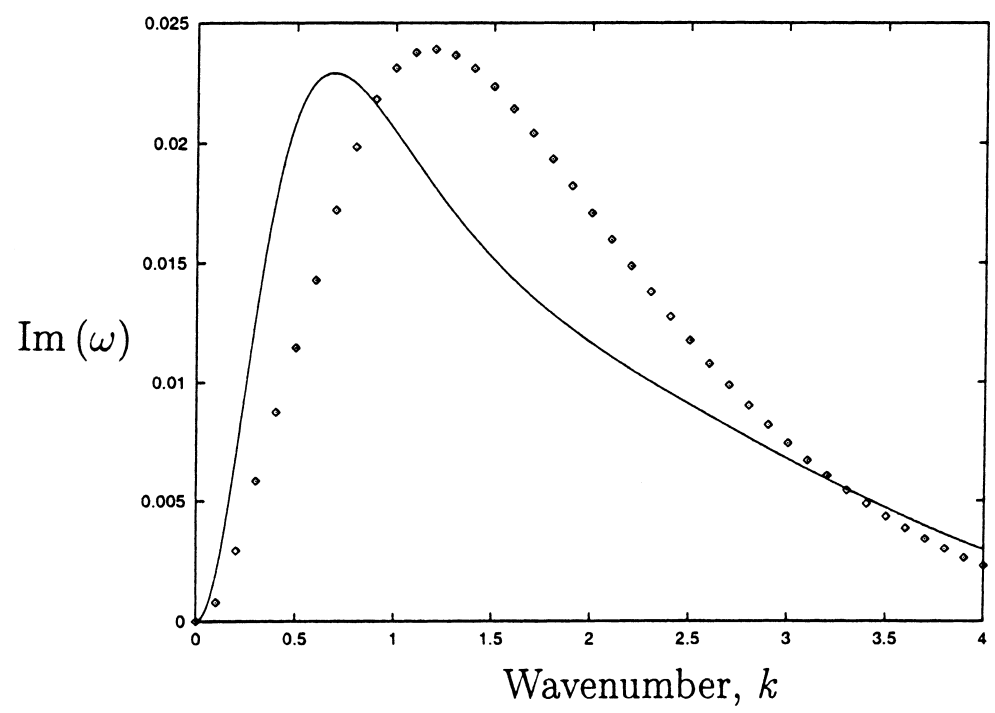

Fig. 6. Plot of growth rate against wavenumber for a representative case in which both sinuous and varicose modes are unstable. $C_{1}=6, C_{2}=2, \kappa=0.5$ and $W=4$. The solid line shows sinuous modes, and the points varicose modes.

the channel width is found to be the most unstable. It is this wavelength which we might expect to survive longest for a 'blurred' interface.

Fig. 8 shows the perturbation flow for the most unstable point on the sinuous plot of Fig. 6. The discontinuity in viscosity produces streamlines for the perturbation flow with discontinuous slope at the interface.

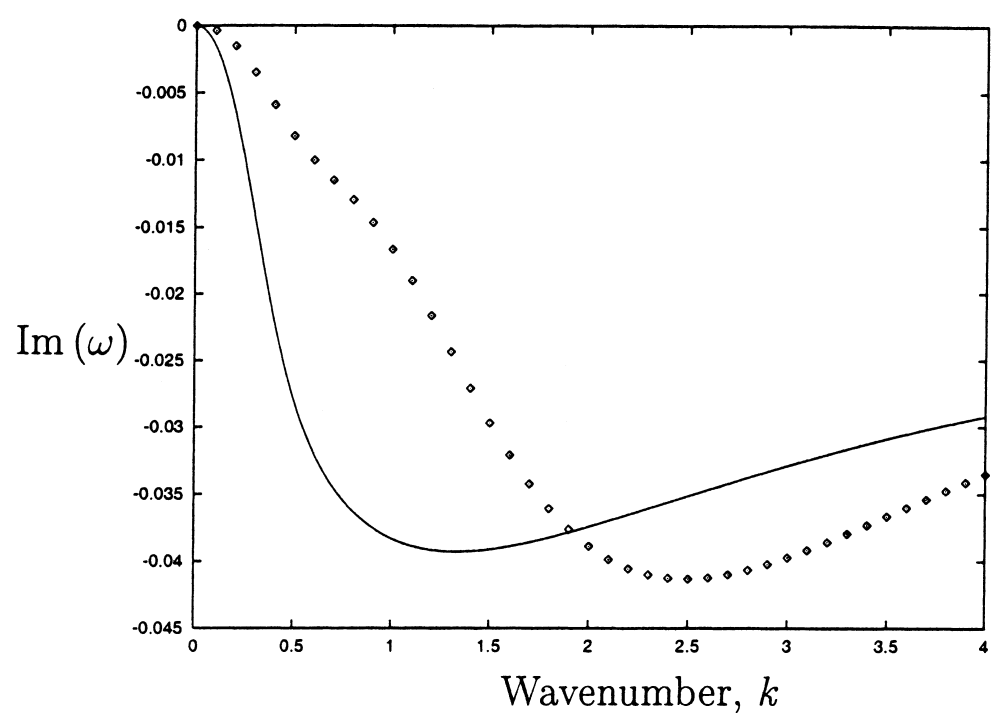

Fig. 7. Plot of growth rate against wavenumber for a representative case in which both sinuous and varicose modes are stable. $C_{1}=2, C_{2}=6, \kappa=0.5$ and $W=4$. The solid line shows sinuous modes, and the points varicose modes. 


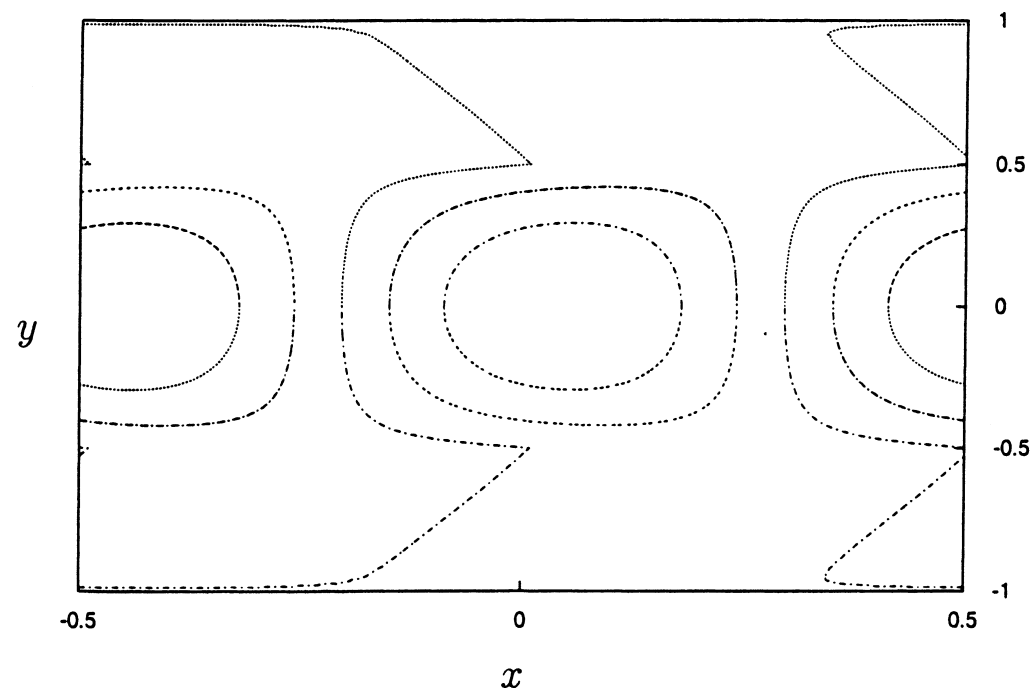

Fig. 8. Streamlines of the perturbation flow, for co-extruded fluids having two different concentrations. The most unstable wavenumber, $k=0.69$, is chosen for a sinuous mode with $C_{1}=6, C_{2}=2$ and $W=4$.

\subsection{Single fluid with continuously varying concentration}

The perturbation equations (39)-(47) for a fluid with continuously stratified concentration are the same as for a single unstratified Oldroyd-B fluid, except for the terms involving $C^{\prime}$. We continue to neglect the term involving $W^{\prime}$.

The connection between these extra terms and their $\delta$-function counterparts for the case of two separate fluids discussed above, is clear: in Eq. (39), the term $-2 \mathrm{i} k W \zeta G^{2} / y^{2}\left(C(1+C)^{-2}\right)^{\prime}$ becomes $-\mathrm{i} k W \zeta\left[\Sigma_{11}\right]$, i.e. it is a jump in $N_{1}$. Similarly, in Eqs. (42),(44) and (45), the term $-\zeta G y C^{\prime}(1+C)^{-2}$ becomes $\zeta\left[U^{\prime}\right]$, i.e. a jump in velocity gradient caused by the jump in viscosity. We now consider what happens when the interface is blurred.

\subsubsection{Specific C-profile}

As an illustration we take $C(\eta)$ as

$$
C(\eta)=\bar{C}+\frac{\Delta C}{\pi} \arctan \{(\eta-\kappa) / q\}
$$

so that the limit $q \rightarrow 0$ is a Heaviside function, equivalent to the two-fluid case with $C_{1}=\bar{C}+\Delta C / 2$ and $C_{2}=\bar{C}-\Delta C / 2$.

For long waves, we can again divide the fluid into two regions: an outer, in which the fluid has the same response as for the interfacial problem, and an inner, in which the variation of $C$ is important. As noted above, the real part of the eigenvalue for the two-fluid case here does not correspond to the interfacial velocity. Nevertheless, the perturbation flow is still driven entirely by forcing at the interface, and so the interface region is central to the dynamics. The scalings are therefore the same as for the problem discussed in Eq. (3). As the 'blurring' is increased, differential advection of different 


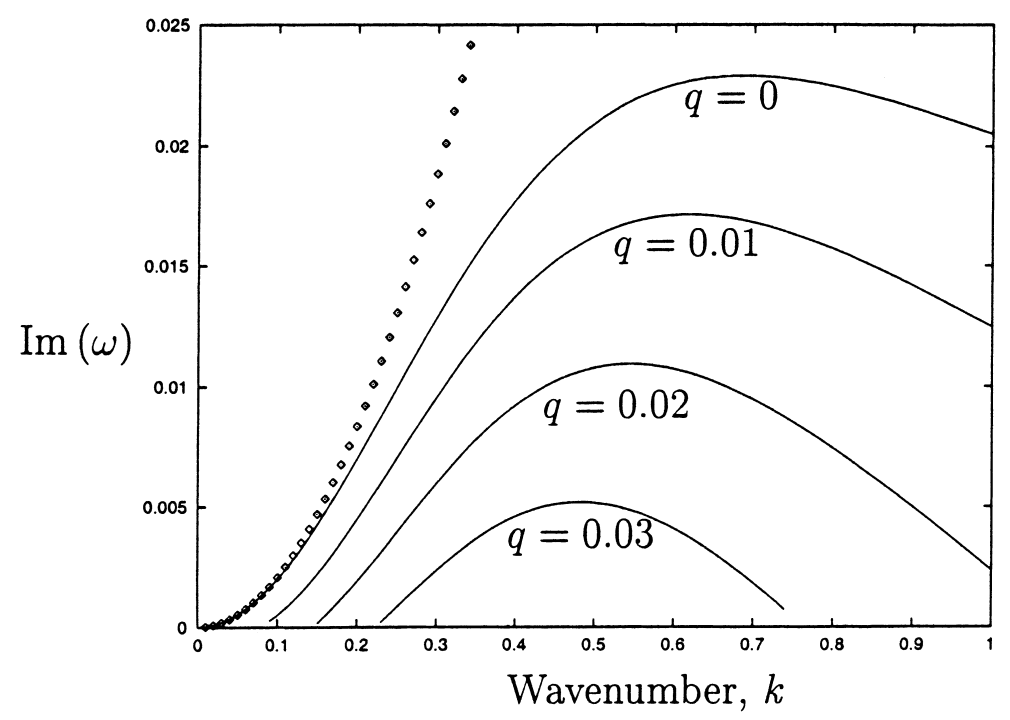

Fig. 9. Growth rate plotted against wavenumber for an increasingly 'blurred' $C$ profile. $W=4, \bar{C}=4, \Delta C=4, \kappa=0.5$. The long-wave asymptotic growth-rate (quadratic in $k$ ) for $q=0$ is shown as a dotted line. At $q=0.04$ the eigenmode has ceased to exist.

layers within the interface causes the forcing due to $N_{1}$ from these different layers to drift out of phase. It follows that no mode can be found for very long waves with $q \gg k$, and that the form of $\sigma$ becomes

$$
\sigma=\sigma_{0}(1-A q / k)
$$

for some constant $A$. For this case the eigenvalue for the two-fluid problem is complicated and we have not found an explicit asymptotic solution for blurred profiles of $C(\eta)$.

A numerical study (of the same form as that in Section 3) reveals that the response to 'blurring' of the interface is as expected (Fig. 9 shows an example). Roots are shown for $q=0,0.01,0.02$ and 0.03 . The mode disappears when $\operatorname{Im}(\omega)=0$. Higher values of $q$ can be found that show instability, by increasing the two-fluid growth rate $\sigma_{0}$, in this case by increasing $W$. However this process cannot continue indefinitely; no roots could be found for $q \geq 0.05$ for any value of $W$.

\section{Stability properties of models with identical velocity and stress profiles}

\subsection{A model problem}

We consider channel flow of two different fluids chosen to have the same velocity and stress profiles. The first is an Oldroyd-B fluid of the type already investigated; the second is a generalisation of a White-Metzner fluid. Our aim is to show that the former is unstable but the latter stable, indicating, as suggested in Section 3.2, that it is the Lagrangian convection of fluid properties that drives the coextrusion instability. 


\subsubsection{A modified White-Metzner fluid}

The White-Metzner fluid, [10], has a relaxation time which is shear-rate dependent. Thus

$$
\begin{aligned}
& \boldsymbol{\sigma}=-p \boldsymbol{I}+\frac{1}{W_{0}} \boldsymbol{A}, \\
& \nabla=-\frac{1}{\tau(\dot{\gamma})}(\boldsymbol{A}-\boldsymbol{I}), \\
& \dot{\boldsymbol{\gamma}}=\sqrt{2 \boldsymbol{E}: \boldsymbol{E}}
\end{aligned}
$$

where $W_{0}$ is a constant.

In channel flow, this fluid exhibits a first normal stress difference $2 W_{0} G^{2} y^{2}$ that is quadratic in distance across the channel. This result is independent of the choice of relaxation function $\tau(\dot{\gamma})$.

In order to compare with a fluid which shows the 'co-extrusion' instability, we need to make a small modification to this model so as to generate a fluid which shows steep gradients in $N_{1}$ across the channel. For this purpose we add a solvent viscosity, which can reduce the effect of $\tau$ on the shear stress, and thus increase its effect on the normal stress. We consider a 'modified' White-Metzner fluid

$$
\begin{aligned}
& \boldsymbol{\sigma}=-p \boldsymbol{I}+2 \boldsymbol{E}+\frac{C_{0}}{W_{0}} \boldsymbol{A}, \\
& \stackrel{\nabla}{\boldsymbol{A}}-\frac{1}{\tau(\dot{\gamma})}(\boldsymbol{A}-\boldsymbol{I}) .
\end{aligned}
$$

In the case $\tau(\dot{\gamma})=W_{0}$ we regain the uniform Oldroyd-B fluid ${ }^{4}$.

The base state for channel flow of our modified White-Metzner fluid is

$$
\begin{aligned}
& \dot{\gamma}_{0}=\left|U^{\prime}\right|=-U^{\prime}, \\
& \tau_{0}=\tau\left(\dot{\gamma}_{0}\right)
\end{aligned}
$$

which then gives

$$
\begin{aligned}
\boldsymbol{A} & =\left(\begin{array}{cc}
1+2 \tau_{0}^{2} U^{\prime 2} & \tau_{0} U^{\prime} \\
\tau_{0} U^{\prime} & 1
\end{array}\right), \\
P & =C_{0} / W_{0}+G x, \\
\Sigma & =\left(\begin{array}{ll}
-G x+2 C_{0} \tau_{0}^{2} U^{\prime 2} / W_{0} & G y \\
G y & -G x
\end{array}\right),
\end{aligned}
$$

\footnotetext{
${ }^{4}$ In the perturbation equations of Section 5.2, we will see that $W_{0}$ only appears as part of $C_{0} / W_{0}$, and so we may without loss of generality set $W_{0}=1$. Physically, this is simply a matter of taking the elastic timescale into the definition of $\tau$, or equivalently using a different non-dimensionalisation for $\boldsymbol{\Sigma}$. However, for easy comparison with the Oldroyd-B fluid it is more convenient to leave both $C_{0}$ and $W_{0}$ in the formulation.
} 
and finally

$$
\left(1+C_{0} \tau_{0} / W_{0}\right) U^{\prime}=G y .
$$

(Eq. (62)) defines $\dot{\gamma}_{0}$, which may be computed numerically for a suitably chosen function $\tau(\dot{\gamma})$.

We now consider an arctan profile for $\tau$

$$
\tau(\dot{\gamma})=\bar{\tau}+\frac{\Delta \tau}{\pi} \arctan \left\{\left(\dot{\gamma}-\dot{\gamma}_{\kappa}\right) / q\right\} .
$$

This gives us a model with parameters $C_{0}, W_{0}, \bar{\tau}, \Delta \tau, q$ and $\dot{\gamma}_{\kappa}$. For small values of $C_{0}$ and $q$, it is capable of giving high gradients of $N_{1}$ because

$$
U^{\prime} \approx G y,
$$

and so

$$
N_{1} \approx 2 C_{0} G^{2} y^{2} \tau(|G y|)^{2} / W_{0} .
$$

We therefore select the specific model parameters: $C_{0}=0.1, q=0.002, W_{0}=1, \bar{\tau}=3.5, \Delta \tau=1$ and $\dot{\gamma}_{\kappa}=1$. Solving Eq. (62) numerically gives $\tau_{0}(y)$ as shown as points in Fig. 10.

\subsubsection{Oldroyd-B fluid}

We now wish to choose the functions $C(\eta)$ and $W(\eta)$ for an Oldroyd fluid so that its base state velocity profile, pressure and stresses closely approximate those of the White-Metzner fluid above.

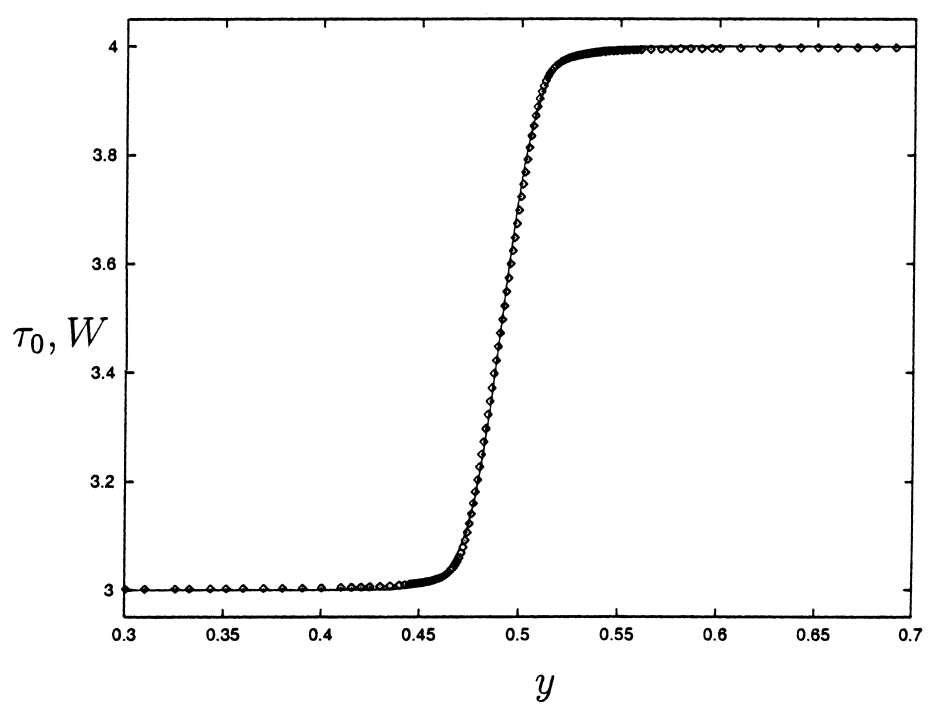

Fig. 10. Profile of $\tau_{0}(y)$ given by Eq. (63) (points) for a White-Metzner fluid; and the function $W(y)$ given by Eq. (64) (solid line) for an Oldroyd-B fluid. 
For the steady state flow of the Oldroyd fluid we have

$$
\begin{aligned}
& \Sigma_{12}=(1+C(y)) U^{\prime}, \\
& N_{1}=2 C W U^{\prime 2}, \\
& P=C / W+G x .
\end{aligned}
$$

Comparing with Eqs. (60)-(62), for equivalence of the base state stress and velocity between the two fluids, we need

$$
C(y)=C_{0} \tau_{0}(\dot{\gamma}) / W_{0}=0.1 W(y),
$$

and

$$
W(y)=\tau_{0}(\dot{\gamma}) .
$$

A closed form solution is not available, but we obtain a good fit (shown in Fig. 10) with

$$
W(y)=3.5+0.5 \tanh \{60(y-0.491)\} .
$$

\subsection{Perturbation equations for the modified White-Metzner fluid}

The perturbation equations for the Oldroyd-B fluid are Eqs. (39)-(47) as previously discussed. The perturbation equations for our modified White-Metzner fluid are

$$
\begin{aligned}
& \mathrm{i} k \sigma_{11}+\sigma_{12}^{\prime}=0, \\
& \mathrm{i} k \sigma_{12}+\sigma_{22}^{\prime}=0, \\
& \sigma_{11}=-p+2 \mathrm{i} k \psi^{\prime}+\frac{C_{0}}{W_{0}} a_{11}, \\
& \sigma_{12}=\psi^{\prime \prime}+k^{2} \psi+\frac{C_{0}}{W_{0}} a_{12}, \\
& \sigma_{22}=-p-2 \mathrm{i} k \psi^{\prime}+\frac{C_{0}}{W_{0}} a_{22}, \\
& \left(-\mathrm{i} \omega+\mathrm{i} k U+\frac{1}{\tau_{0}}\right) a_{11}=\mathrm{i} k \psi A_{11}^{\prime}+2 A_{12} \psi^{\prime \prime}+2 A_{11} i k \psi^{\prime}+2 U^{\prime} a_{12}+\frac{\tau_{1}}{\tau_{0}^{2}}\left(A_{11}-1\right), \\
& \left(-\mathrm{i} \omega+\mathrm{i} k U+\frac{1}{\tau_{0}}\right) a_{12}=\mathrm{i} k \psi A_{12}^{\prime}+\psi^{\prime \prime}+A_{11} k^{2} \psi+U^{\prime} a_{22}+\frac{\tau_{1}}{\tau_{0}^{2}} A_{12}, \\
& \left(-\mathrm{i} \omega+\mathrm{i} k U+\frac{1}{\tau_{0}}\right) a_{22}=-2 \mathrm{i} k \psi^{\prime}+2 A_{12} k^{2} \psi .
\end{aligned}
$$

In these equations, $\tau_{1}$ is the linear correction to the time 'constant' reflecting the fact that a material particle responds instantaneously to the local shear-rate. It is given as

$$
\tau_{1}=\dot{\gamma}_{1} \partial \tau_{0} / \partial \dot{\gamma}_{0}
$$


where

$$
\dot{\gamma}_{1}=-\left(\psi^{\prime \prime}+k^{2} \psi\right)
$$

It is not helpful to use the alternative form of the equations described in Section 2.4, because the perturbation to $\tau$

$$
\tau_{1}=-\left.\left(\psi+k^{2} \psi\right)(\partial \tau / \partial \dot{\gamma})\right|_{\dot{\gamma}=0}
$$

does not depend on the streamline displacement, $\zeta$, but rather on the perturbed flow itself. Our splitting was based on the observation that part of the perturbation was due to the advection of base state quantities by the streamline displacement. Where this advection does not occur, there is no simplification.

The Eqs. (65)-(74) may be solved numerically in the same way as those in Section 2.

\subsection{Numerical results}

The two fluids we have chosen appear identical in terms of their base-flow velocities and stresses. Figs. 11 and 12 show part of their stability spectra to varicose disturbances. (For each fluid, the only modes not shown are more stable than those shown, and may safely be ignored.) The Oldroyd fluid has an unstable mode for moderate values of $k$, as discussed in Sections 3 and 4. No such mode exists for the White-Metzner fluid, which is stable to perturbations of all wavenumbers. This confirms our speculation that advection of material properties is a crucial part of the co-extrusion instability mechanism.

The stable parts of the spectra are very similar (as shown in the close-up in Fig. 12). This indicates that the differences between the models only affect the 'co-extrusion' mode, and not the behaviour of the stable modes of both fluids.

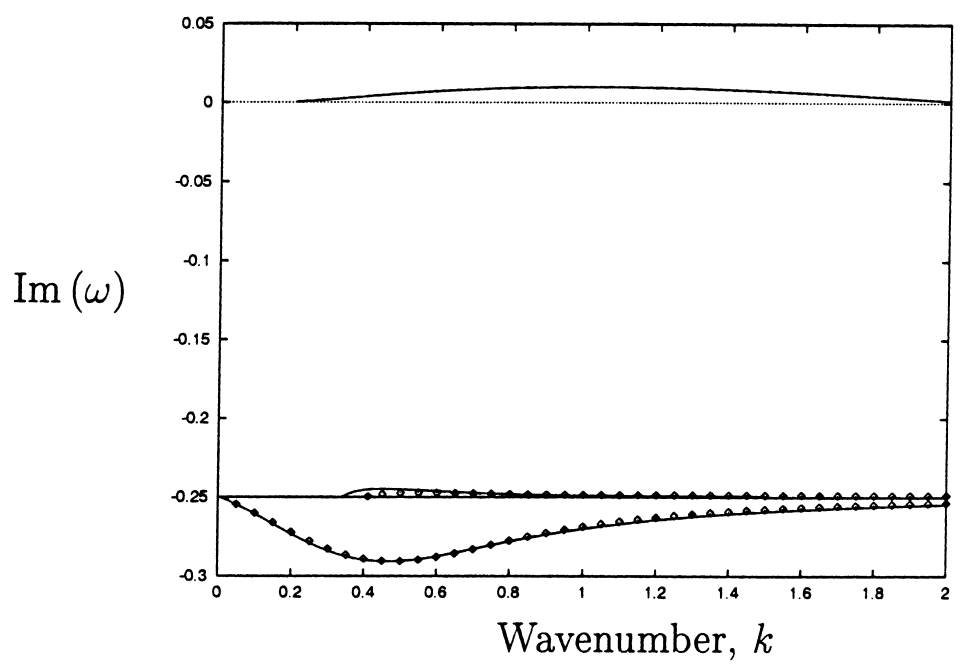

Fig. 11. The least stable part of the spectra of the generalised fluids. The solid lines are for the Oldroyd fluid (the horizontal line $\operatorname{Im}(\omega)=-0.25$ is part of its continuous spectrum), and the points are for the modified White-Metzner fluid. Only the Oldroyd fluid shows the unstable mode. 


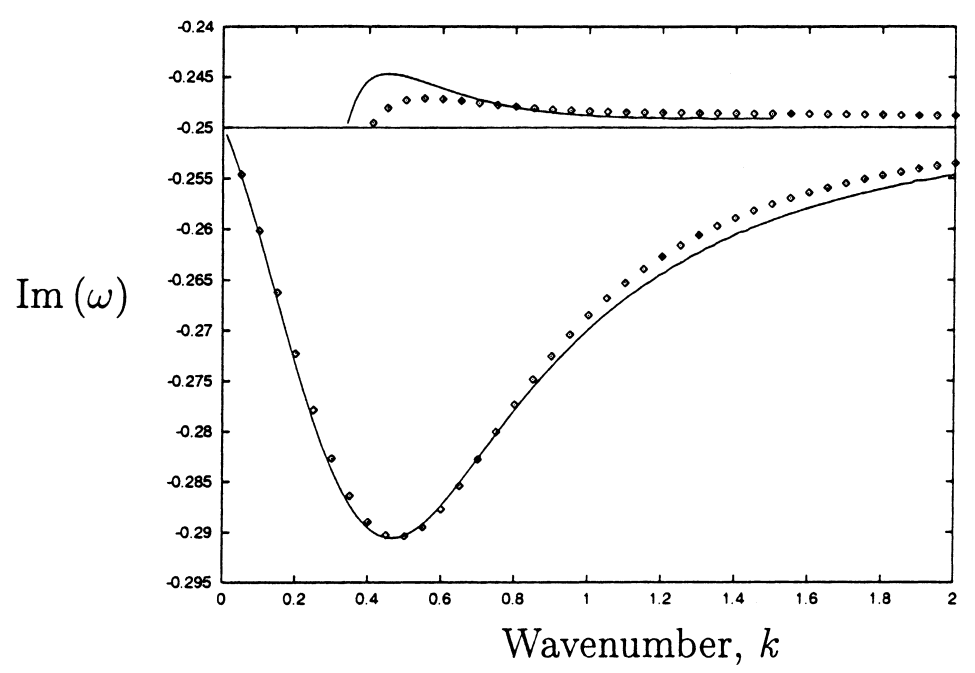

Fig. 12. A close-up of the lower region of Fig. 11, demonstrating the close correspondence between the two spectra.

\section{Conclusions}

We have shown that channel flow of a single stratified Oldroyd-B fluid can undergo a purely elastic 'co-extrusion' instability provided that its normal stress coefficient varies steeply enough, and that the analogous two-fluid extrusion problem is also unstable. The convection of fluid properties is a crucial element of this instability: a modified White-Metzner fluid having the same stress and velocity profiles is stable.

Where both sinuous and varicose disturbances are unstable, sinuous modes are generally more unstable, and it is disturbances that have wavelengths comparable with the channel width that grow fastest on linear theory and are also the most long-lasting as the interface is 'blurred'.

The mechanism that inhibits the instability as the degree of 'blurring' of the interface is increased is relative convection of different fluid layers that generates destructive interference as the disturbances to different layers drift out of phase.

We find qualitatively similar results for both a constant-viscosity fluid in which the relaxation time of polymers varies across the flow (which is analytically more tractable) and for a varying-viscosity fluid in which the relaxation-time is uniform, but the concentration of polymers changes across the channel.

We are not aware of any experiment in which the concentration of polymer across a channel has been deliberately varied. Such experiments would be of interest. Our calculation suggests that steep concentration gradients must be included if our instability is to arise; concentration gradients due to imperfect upstream mixing are unlikely to be sufficient.

The analysis of Section 4 has a bearing on a proposed mechanism for the sharkskin instability. Chen and Joseph [11] suggest that the high stresses near the wall can cause polymer molecules to migrate inwards, creating a depleted wall region. They then propose that the interface dividing this region from the bulk of the fluid would be subject to a short-wave interfacial instability of the type discussed in $[7,8]$. Our analysis shows that a thin outer layer with lower viscosity than the bulk will be stable to long waves in the absence of inertia, and that a small amount of 'blurring' of the interface will stabilise short 
waves. An interface created by migration will never be truly sharp, and therefore the purely elastic instability mechanism they propose could not occur in practice.

In order to observe the coextension instability, we require that the material properties be advected with the flow. It follows that the existence of an instability is not dictated by the base state alone, but depends also on the constitutive behaviour. This has important implications for constitutive modelling. The Oldroyd-B and White-Metzner fluids may be considered as extreme cases. For Oldroyd-B, a material parcel retains its relaxation time throughout its life. Therefore the timescale on which this changes for any fluid parcel is effectively infinite. For a White-Metzner fluid, $\tau=\tau(\dot{\gamma})$, i.e. a parcel's time constant is an instantaneous function of the applied rate of strain. $\tau$ has no memory of past values, but rather reacts to the flow on an instantaneous timescale.

The stability characteristics are evidently affected by the existence of microscopic mechanisms for modifying (or 'diffusing') material properties, and these cannot be inferred from base-state quantities alone.

\section{Acknowledgements}

This work was assisted by the EU Human Capital and Mobility Programme grant number CHRXLT93-0200. HJW was supported by a Research Studentship from the EPSRC.

\section{References}

[1] G.H. McKinley, P. Pakdel, A Öztekin, Rheological and geometric scaling of purely elastic flow instabilities, J. NonNewtonian Fluid Mech. 67 (1996) 19-47.

[2] K.P. Chen, Interfacial instability due to elastic stratification in concentric coextrusion of two viscoelastic fluids, J. NonNewtonian Fluid Mech. 40(2) (1991) 155-176.

[3] E.J. Hinch, O.J. Harris, J.M. Rallison, The instability mechanism for two elastic liquids being co-extruded, J. NonNewtonian Fluid Mech. 43(2-3) (1992) 311-324.

[4] T.C. Ho, M.M. Denn, Stability of plane Poiseuille flow of a highly elastic liquid, J. Non-Newtonian Fluid Mech. 3(2) (1978) 179-195.

[5] E.S.G. Shaqfeh, Purely elastic instabilities in viscometric flows, Ann. Rev. Fluid Mech. 28 (1996) $129-186$.

[6] H.J. Wilson, Shear Flow Instabilities in Viscoelastic Fluids, PhD thesis, University of Cambridge, 1998.

[7] H.J. Wilson, J.M. Rallison, Short wave instability of co-extruded elastic liquids with matched viscosities, J. NonNewtonian Fluid Mech. 72(2-3) (1997) 237-251.

[8] K.P. Chen, D.D. Joseph, Elastic short-wave instability in extrusion flows of viscoelastic liquids, J. Non-Newtonian Fluid Mech. 42(1-2) (1992) 189-211.

[9] B.W. Char, K.O. Geddes, G.H. Gonnet, B.L. Leong, M.B. Monagan, S.M. Watt. Maple V Library/Language Reference Mannual. Springer, Waterloo Maple Publishing, 1991.

[10] R.G. Larson, Constitutive equations for polymer melts and solutions, Butterworths, Boston, MA, 1988.

[11] K.P. Chen, D.D. Joseph, Long-wave and lubrication theories for core-annular flow, Phys. Fluids A 3(11) (1991) 26722679. 